\title{
Improved Piglet Performance and Reduced Mortality and Antimicrobial use Following Oral Vaccination with a Live Non-Pathogenic Escherichia coli F4/ F18 Vaccine Against Post-Weaning Diarrhoea
}

\author{
Vangroenweghe $\mathrm{F}^{1,2 *}$ \\ ${ }^{1}$ BU Food Animals, Plantijn en Moretuslei 1A, 2018 \\ Antwerpen, Belgium \\ ${ }^{2}$ Ghent University, Faculty of Veterinary Medicine, Unit \\ of Porcine Health Management, Merelbeke, Belgium \\ *Correspondling author: Frédéric Vangroenweghe, BU \\ Food Animals, Elanco Benelux, Plantijn en Moretuslei 1A, \\ 2018 Antwerpen, Belgium
}

Received: May 03, 2021; Accepted: May 22, 2021;

Published: May 29, 2021

\begin{abstract}
Background: Post-Weaning Diarrhoea (PWD) in pigs is a worldwide economically important disease, which is frequently controlled using antimicrobials. However, emergence of antimicrobial resistance in E. coli strains and new EU regulations urge the need for alternative control measures, such as adapted feeding strategies or immunization. Oral vaccination of suckling piglets using a live non-pathogenic $E$. coli F4/F18 vaccine was performed in 10 farrow-to-finish sow farms to prevent against post-weaning diarrhoea due to F4-Enterotoxigenic E. coli (ETEC) or F18-ETEC. The vaccination strategy was compared to the standard therapeutic approach in each farm, meanwhile collecting data on Average Daily Weight Gain (ADWG), Feed Conversion Rate (FCR), mortality rate and treatment incidence with antimicrobial drugs (TI100) during the post-weaning period.
\end{abstract}

Results: Vaccine-treated groups demonstrated a significant improvement in FCR, mortality rate and TI100 as compared to the Control group. The ADWG only marginally and non-significantly improved in the Vaccine-treated group.

Conclusions: In conclusion, the present study demonstrated the efficacy of an oral live non-pathogenic E. coli F4/F18 vaccine (Coliprotec ${ }^{\circledR}$ F4/F8; Elanco Animal Health) for active immunization of piglets against PWD due to F4-ETEC and F18-ETEC under field conditions. For several economically important performance parameters, such as FCR, mortality rate and TI100, E. coli vaccination performed significantly better as compared to the standard therapeutic approach. Therefore, vaccination against PWD due to F4-ETEC or F18-ETEC using an oral live non-pathogenic E. coli F4/F18 vaccinated may be considered a good alternative to consolidate post-weaning piglet performance results while meeting the new European requirements concerning prudent use of antimicrobials in intensive pig production.

Keywords: F4-ETEC; F18-ETEC; PWD; E. coli F4/F18 vaccine Performance; Antimicrobial reduction

\section{Abbreviations}

ADWG: Average Daily Weight Gain; FCR: Feed Conversion Rate; BMS: Batch-Management System; CVMP: Committee for Veterinary Medicinal Products; ETEC: Enterotoxigenic Escherichia coli; EU: European Union; LT: Heat-Labile Toxin; MCFAs: Medium Chain Fatty Acids; ppm: Parts Per Million; PWD: Post-Weaning Diarrhoea; STa: Heat-Stabile Toxin a; STb: Heat-Stabile Toxin b; Stx2e: ShigaToxin 2e; ZnO: Zinc Oxide

\section{Introduction}

Post-Weaning Diarrhoea (PWD) in pigs is globally considered to be the most important economic disease [1]. It is characterized by an increase in mortality, significant weight losses and growth retardation, in combination with increased treatment costs, higher use of antimicrobials and more pronounced batch variation [2-
8]. Enterotoxigenic E. coli (ETEC) has been shown to be the most important cause of PWD $[9,10]$. The ETEC pathotype is typically characterized by two types of virulence factors: fimbrial adhesins, which mediate attachment to porcine intestinal enterocytes, and enterotoxins, which disrupt fluid homeostasis in the small intestine. This results in mild to severe diarrhoea within a few days postweaning, associated with clinical signs of dehydration, loss of body condition (= disappearance of muscle volume) and mortality [1]. The adhesive fimbriae most commonly occurring in ETEC from pigs with PWD are F4 (K88) and F18 [9-11]. Other fimbriae such as F5 (K99), F6 (987P) and F41 rarely occur in E. coli isolates from PWD [9-14]. The main enterotoxins associated with ETEC in pigs are Heat-Labile Toxin (LT), Heat-Stable Toxin a (STa) and Heat-Stable Toxin b (STb). In some exceptional cases, both enterotoxins and a Shiga toxin (Stx2e) are produced by the pathogenic strains [9-11].

Clinical disease is predominantly controlled by antimicrobials,
Austin J Infect Dis - Volume 8 Issue 2 - 2021

Submit your Manuscript | www.austinpublishinggroup.com

Vangroenweghe. (C) All rights are reserved
Citation: Vangroenweghe F. Improved Piglet Performance and Reduced Mortality and Antimicrobial use Following Oral Vaccination with a Live Non-Pathogenic Escherichia coli F4/F18 Vaccine Against Post-Weaning Diarrhoea. Austin J Infect Dis. 2021; 8(2): 1048. 
although emergence of antimicrobial resistance in E. coli strains isolated from clinical cases of PWD indicates an urgent need for alternative control strategies [15-19].

In the light of these developments, many alternative control strategies have recently been explored to increase intestinal health and decrease incidence of PWD due to E. coli in post-weaned piglets [20-22]. Briefly, several feed adaptations have been studied, including addition of dietary fiber, reduction of crude protein levels, changes in feed consistency and feed particle size, supplementation of prebiotics, probiotics and Medium Chain Fatty Acids (MCFA) towards their effect on incidence and severity of PWD and gut health in general. From the late 1980's onwards, several studies on zinc supply to post-weaned piglets have been performed. Several nutritional studies demonstrated the effects of dietary Zinc Oxide $(\mathrm{ZnO})$ in the prevention and healing of PWD [23]. Therefore, $\mathrm{ZnO}$ has been admitted in the prevention and control of PWD at levels up to 3,000 parts per million (ppm) through the feed for a maximum of 14 days post-weaning. However, the Committee for Veterinary Medicinal Products (CVMP) has recently decided that the use of $\mathrm{ZnO}$ in postweaning diets should be phased out the latest by 2022 throughout the EU [24].

Therefore, other preventive strategies have recently been explored $[1,25]$. For an E. coli vaccination against PWD due to F4-ETEC and F18-ETEC, the prerequisite is that active mucosal immunity against F4 and F18 is mounted. This implies the local production of F4- and/ or F18-specific sIgA antibodies, which prevent pathogenic F4-ETEC and F18-ETEC to attach to the intestinal F4- and F18-receptors and thus reduce clinical signs of PWD [25]. Recently, vaccination with a live non-pathogenic E. coli F4, or E. coli F4 and F18 vaccine has demonstrated efficacy against PWD due to F4-ETEC, and F4-ETEC and F18-ETEC [26,27]. Immunization against the F4-ETEC and F18-ETEC pathogens resulted in decreased severity $[26,27,29,30]$ and duration of PWD clinical signs and fecal shedding of F4ETEC and F18-ETEC [26,27]. Moreover, increased weight gain was demonstrated in piglets vaccinated with $E$. coli $\mathrm{F} 4$ vaccine $[26,29,30]$.

Here, we report the results from 10 field trials demonstrating the efficacy of an oral live non-pathogenic E. coli F4/F18 vaccine (Coliprotec F4/F18; Elanco; Greenfield, IN) for active immunization of piglets against PWD caused by F4-ETEC and F18-ETEC under field conditions. In each field trial, Vaccine-treated piglets were compared to Control piglets treated according to the specific standard on-farm treatment protocol.

\section{Materials and Methods}

\section{Farm description}

The field trials were performed from April 2018 until December 2020 on several conventional farrow-to-finish pig farms $(n=10)$ with a variable number of sows (160-1200 sows per farm) in Belgium and the Netherlands. A detailed description of the different farms regarding number of sows on-farm, type of Batch Management System (BMS), E. coli pathotype diagnosis, piglet vaccination age, piglet weaning age, number of piglets included in both Control and Vaccine-treated group and the duration of the post-weaning observation period is given in Table 1. Briefly, the sow farms were managed in a 4 -week BMS (40\%), or a 3-week BMS (40\%). In only 1 farm the sows were managed in a 1-week BMS (10\%) or a 5-week BMS (10\%). The average number of sows on-farm was $611( \pm 112$; min. 160, max. 1,200). Etiological diagnosis of the E. coli pathotype was performed as previously described [10]. In total, 6 farms were positive for F18-ETEC, whereas the other 4 farms were F4-ETEC positive. Throughout the different field trials, 49,936 and 46,929 piglets were included in the Control and Vaccine-treated group, respectively. The average age of piglets at vaccination was 19.30 ( \pm 0.45 days (min. 18; max. 21) and average age of piglets at weaning was $23.20( \pm 0.74)$ days (min. 18; max. 25). Following weaning, piglets were housed in specifically equipped post-weaning facilities, where they were raised for a period of $48.50( \pm 0.82)$ days (min. 42 ; max. 50) on average. The post-weaning facilities were equipped with pens, which could each house between 12 and 30 post-weaned piglets depending on the specific farm. The pens were equipped with slatted plastic floors and room temperature was kept between 27 and $31^{\circ} \mathrm{C}$ for the first 2-3 weeks post-weaning. All post-weaning facilities were equipped with mechanical ventilation. Post-weaning facilities were cleaned and disinfected between subsequent batches.

\section{Experimental design and vaccination with a live non- pathogenic E. coli F4/F18 vaccine}

The live non-pathogenic E. coli F4/F18 vaccine (Coliprotec F4/ F18; Elanco, Greenfield, IA, USA) has a rapid onset of immunity (7 days) and a duration of immunity of 21 days post-vaccination, which covers the most critical period of PWD [1]. An efficacy trial using an experimental E. coli $\mathrm{F} 4$, or E. coli $\mathrm{F} 4$ and/or F18 challenge at 3 days post-weaning showed reduction of the severity and duration of PWD and reduction in fecal shedding of pathogenic F4-ETEC and F18ETEC $[26,27]$. Therefore, piglets were vaccinated from 18 days of age onwards during the suckling period to mount sufficient protective local immunity in the gut at the moment of onset of clinical signs of PWD [33].

In most farms, a historical Control group, immediately preceding the introduction of $E$. coli $\mathrm{F} 4 / \mathrm{F} 18$ vaccination, was assigned to compare to the Vaccine-treated group. However, in farm 3 and 5, concurrent Control and Vaccine-treated groups were run in the field trials. In case of a concurrent trial, sows were randomly assigned to the Vaccine-treated or Control group based on their parity and sow number. Parities were equally distributed to both treatment groups. Piglets from sows assigned to the Vaccine-treated group were vaccinated orally through drenching with $2 \mathrm{ml}$ of a live nonpathogenic E. coli $\mathrm{F} 4 / \mathrm{F} 18$ vaccine according to the manufacturer's specifications. Piglets from sows in the Control group were not treated nor vaccinated. No antimicrobials were administered to piglets from 15 days of age onwards to omit interference with development of protective local immunity by the E. coli $\mathrm{F} 4 / \mathrm{F} 18$ vaccine during the 7 days following vaccination

\section{Feeding strategies and treatment}

Farm-specific feeding strategies were continued in both Control and Vaccine-treated group, with the only exception that inclusion of $\mathrm{ZnO}$ was discontinued in both treatment groups. Depending on the farm, 1-phase, 2-phase of 3-phase feeding schedules were used throughout the post-weaning period.

No standard group treatments were performed during the entire study period. Individual piglets with severe clinical signs of PWD 
Table 1: Descriptive data from different field trials using a live, non-pathogenic, oral E. coli F4/F18 vaccine in piglets to prevent clinical impact of post-weaning diarrhea due to E. coli. Included data are related to farm identification, number of sows, type of Batch-Management System (BMS), diagnosis of E. coli pathotype (F4-ETEC or F18-ETEC), piglet vaccination age and piglet weaning age, number of piglets included in the trial (control/vaccinated) and duration of the post-weaning observation period.

\begin{tabular}{|c|c|c|c|c|c|c|c|}
\hline Farm ID & \# sows on-farm & BMS type & Pathotype diagnosis & Piglet vaccination age & Piglet weaning age & \# piglets included & $\begin{array}{c}\text { Duration post-weaning } \\
\text { period }\end{array}$ \\
\hline 1 & 600 & $4 \mathrm{WS}$ & F18-ETEC & 18 & 21 & $1800 / 3000$ & 50 \\
\hline 2 & 600 & 4WS & F4-ETEC & 18 & 21 & $17000 / 16500$ & 50 \\
\hline 3 & 700 & $4 \mathrm{WS}$ & F18-ETEC & 21 & 21 & 4341/3039 & 50 \\
\hline 4 & 450 & $3 W S$ & F4-ETEC & 18 & 25 & $1840 / 1910$ & 47 \\
\hline 5 & 250 & $3 W S$ & F4-ETEC & 20 & 25 & $610 / 600$ & 42 \\
\hline 6 & 800 & $4 \mathrm{WS}$ & F18-ETEC & 18 & 21 & $7880 / 8000$ & 49 \\
\hline 7 & 250 & $3 W S$ & F18-ETEC & 20 & 25 & $900 / 1200$ & 50 \\
\hline 8 & 1100 & $5 \mathrm{WS}$ & F18-ETEC & 18 & 21 & $12839 / 10140$ & 47 \\
\hline 9 & 160 & $3 W S$ & F4-ETEC & 21 & 26 & $876 / 910$ & 50 \\
\hline 10 & 1200 & $1 \mathrm{WS}$ & F18-ETEC & 21 & 26 & $1850 / 1630$ & 50 \\
\hline
\end{tabular}

were treated with an injectable antimicrobial, i.e. lincomycin. Other disorders were treated by the farmer, following consultation of the veterinarian, with the appropriate antimicrobial where needed. All individual treatments were registered on a daily basis.

\section{Performance parameters}

The following performance parameters were collected during the field trials: piglet weight at the start and end of the post-weaning period, feed intake during the post-weaning period, number of dead piglets and individual treatments with antimicrobials. Average Daily Weight Gain (ADWG) $(n=7)$ was calculated based on piglet weight and number of days under observation in the post-weaning facility. Feed Conversion Rate (FCR) $(\mathrm{n}=4)$ the amount of feed to add one kg of bodyweight, was calculated based on average daily weight gain and feed intake during the post-weaning period. Mortality rate $(n=10)$ was calculated based on number of dead piglets over the total number of piglets included in every specific field trial. Treatment incidence 100 (TI100) $(n=9)$ was calculated based on the number of individual injections per treatment for a total of 100 piglets over a calculated period of 100 days.

\section{Statistical analysis}

For all continuous data, effect of treatment was assessed using JMP 15.0 with pairwise comparison using $t$-test with pooled standard deviations. All tests were performed at the nominal level of $5 \%$.

\section{Results}

A summary of all obtained trial results is given in Table 2.

\section{Average daily weight gain and feed conversion rate}

Average daily weight gain was not significantly higher (359.29 \pm $15.83 \mathrm{~g} / \mathrm{d} ; \mathrm{P}=0.1641$ ) in the Vaccine-treated group as compared to the Control group $(348.14 \pm 10.25 \mathrm{~g} / \mathrm{d}$ ) (Figure 1). A broad range of ADWG values could be observed between the different field trials varying from 304 to $384 \mathrm{~g} / \mathrm{d}$ in the Control group and from 307 to $420 \mathrm{~g} / \mathrm{d}$ in the Vaccine-treated group.

Feed conversion rate was significantly lower $(1.66 \pm 0.09 \mathrm{~kg}$ feed $/$ $\mathrm{kg}$ gain; $\mathrm{P}=0.0164)$ in the Vaccine-treated group as compared to the Control group (1.72 $\pm 0.10 \mathrm{~kg}$ feed $/ \mathrm{kg}$ gain) (Figure 2). Again, a broad range of FCR values could be observed between the different field
Table 2: Performance results from different field trials using a live, nonpathogenic, oral E. coli F4/F18 vaccine in piglets to prevent clinical impact of postweaning diarrhea due to $E$. coli. Included data are related to treatment (contro vs. vaccination), Average Daily Weight Gain (ADWG, grams per day), Feed Conversion Rate (FCR, kg feed per kg weight gain), mortality rate (percentage) and treatment incidence per 100 days in production (TI100, days). N/A: No applicable when no data are available for that specific parameter.

\begin{tabular}{|c|c|c|c|c|c|}
\hline Farm ID & Treatment & ADWG & FCR & Mortality rate & $\mathrm{TI}_{100}$ \\
\hline \multirow{2}{*}{1} & Control & N/A & N/A & 6 & 54.7 \\
\hline & Vaccination & N/A & N/A & 3 & 15.6 \\
\hline \multirow{2}{*}{2} & Control & 384 & 1.91 & 3.5 & $\mathrm{~N} / \mathrm{A}$ \\
\hline & Vaccination & 390 & 1.81 & 2.6 & $\mathrm{~N} / \mathrm{A}$ \\
\hline \multirow{2}{*}{3} & Control & 351 & 1.61 & 3.15 & 10.1 \\
\hline & Vaccination & 373 & 1.55 & 1.65 & 1 \\
\hline \multirow{2}{*}{4} & Control & 358 & 1.88 & 3.31 & 6.3 \\
\hline & Vaccination & 420 & 1.83 & 2.3 & 0 \\
\hline \multirow{2}{*}{5} & Control & 329 & N/A & 2.13 & 16.7 \\
\hline & Vaccination & 328 & N/A & 1.4 & 0 \\
\hline \multirow{2}{*}{6} & Control & 320 & N/A & 4.3 & 22 \\
\hline & Vaccination & 318 & N/A & 1.9 & 4.4 \\
\hline \multirow{2}{*}{7} & Control & 304 & N/A & 0.9 & 14.3 \\
\hline & Vaccination & 307 & N/A & 0.9 & 0.7 \\
\hline \multirow{2}{*}{8} & Control & 362 & 1.47 & 3.56 & 10 \\
\hline & Vaccination & 378 & 1.49 & 1.67 & 0 \\
\hline \multirow{2}{*}{9} & Control & N/A & N/A & 11.21 & 18.6 \\
\hline & Vaccination & $\mathrm{N} / \mathrm{A}$ & N/A & 4.51 & 2.4 \\
\hline \multirow{2}{*}{10} & Control & $\mathrm{N} / \mathrm{A}$ & N/A & 2.05 & 7 \\
\hline & Vaccination & N/A & $\mathrm{N} / \mathrm{A}$ & 1.96 & 0 \\
\hline
\end{tabular}

trials varying from 1.49 to $1.91 \mathrm{~kg}$ feed $/ \mathrm{kg}$ gain in the Control group and from 1.47 to $1.83 \mathrm{~kg}$ feed $/ \mathrm{kg}$ gain in the Vaccine-treated group.

\section{Mortality}

Mortality rate was significantly lower $(2.33 \pm 0.42 \% ; \mathrm{P}=0.0083)$ in the Vaccine-treated group as compared to the Control group (5.43 \pm $1.39 \%$ ) (Figure 3). A broad range in mortality rate could be observed between the different field trials varying from 0.90 to $11.21 \%$ in the 


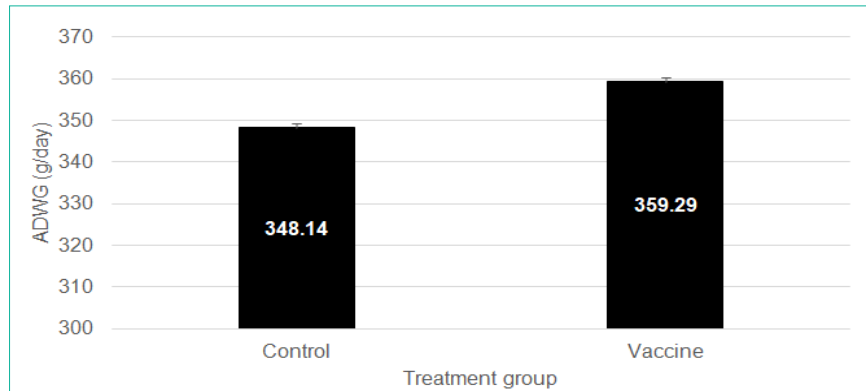

Figure 1: Average daily weight gain (ADGW; mean \pm SEM; expressed as grams per day) in Control and Vaccine-treated animals from different field trials using a live, non-pathogenic, oral E. coli F4/F18 vaccine in piglets to prevent clinical impact of post-weaning diarrhea due to $E$. coli. Data were not significantly different $(P=0.1641)$.

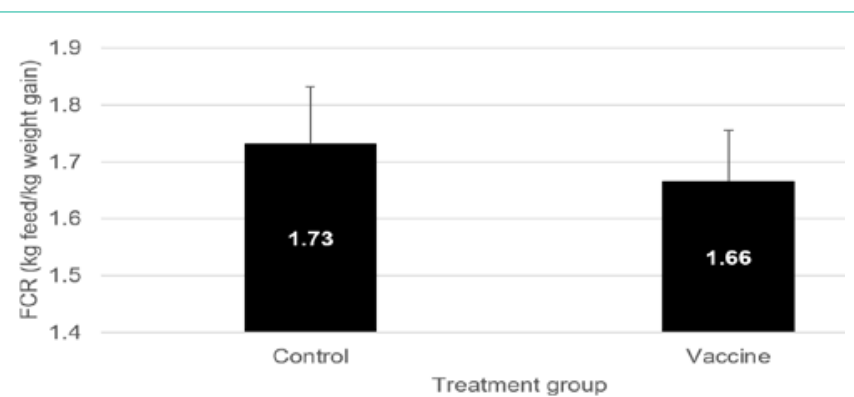

Figure 2: Feed conversion rate (FCR; mean \pm SEM; expressed as kilograms of feed per kilogram of weight gain) in Control and Vaccine-treated animals from different field trials using a live, non-pathogenic, oral E. coli F4/F18 vaccine in piglets to prevent clinical impact of post-weaning diarrhea due to E. coli. Data were significantly different $(P=0.0164)$.

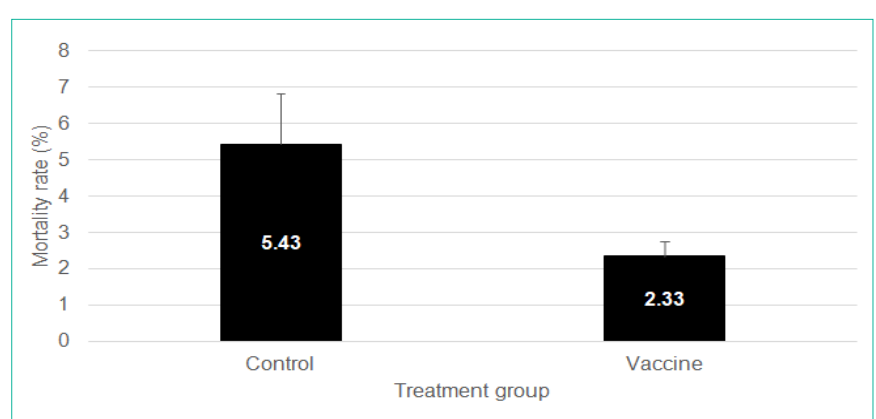

Figure 3: Mortality rate (mean \pm SEM; expressed as percentage) in Control and Vaccine-treated animals from different field trials using a live, nonpathogenic, oral $E$. coli $\mathrm{F} 4 / \mathrm{F} 18$ vaccine in piglets to prevent clinical impact of post-weaning diarrhea due to $E$. coli. Data were significantly different ( $P$ $=0.0083$ ).

Control group and from 0.90 to $4.51 \%$ in the Vaccine-treated group.

\section{Treatment incidence 100}

TI100 was calculated as the total number of individual treatments per 100 piglets per group over a calculated 100-day treatment period. Treatment incidence 100 was significantly lower ( $2.68 \pm 1.69$ days; $\mathrm{P}=0.0009)$ in the Vaccine-treated group as compared to the Control group $(17.74 \pm 4.95)$ (Figure 4). A broad range in TI100 values could be observed between the different field trials varying from 6.30 to 54.7 days in the Control group and from 0.00 to 15.6 days in the Vaccinetreated group.

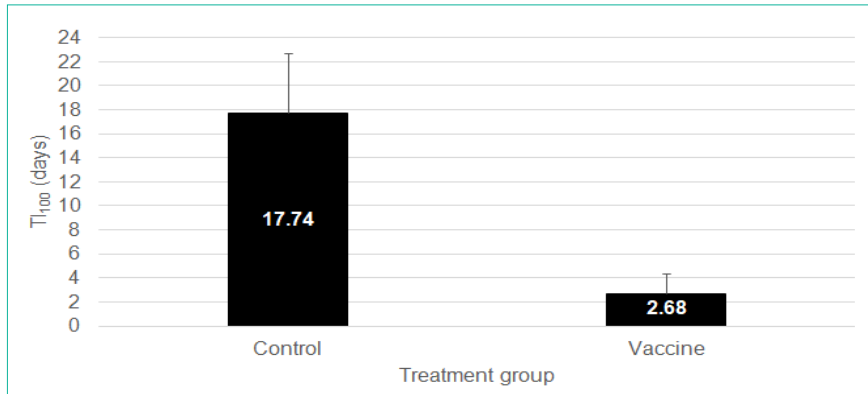

Figure 4: Treatment incidence 100 (TI100; mean \pm SEM; expressed as calculated number of treatment days per 100 days in production) in Control and Vaccine-treated animals from different field trials using a live, nonpathogenic, oral E. coli F4/F18 vaccine in piglets to prevent clinical impact of post-weaning diarrhea due to $E$. coli. Data were significantly different $(P=0.0009)$.

\section{Discussion}

From the current study results, we can conclude that active immunization of piglets against PWD caused by F4-ETEC or F18ETEC resulted in significant better post-weaning piglet performances as compared to the piglets managed under the standard therapeutic approach. Several economically important performance parameters, such as FCR, mortality rate and treatment incidence were significantly improved in the Vaccine-treated as compared to the Control group.

For FCR, an improvement of 0.06 in piglet performance resulted in an approximate economic advantage of about 3.7 eurocent per $\mathrm{kg}$ gain - at the current average market value of 620 euro per tonne of post-weaning piglet feed - or $1.05 \mathrm{~kg}$ less feed per piglet for the same post-weaning weight gain. Considering that feed cost is one of the most important aspects in production, this may already have a significant impact on the net farm's income during the post-weaning phase.

Although in some trials ADWG in the Control group was close to or higher than the ADWG in the Vaccine-treated group, overall we could report a non-significant 11-gram higher ADWG throughout the different field trials. With a post-weaning period of 48.5 days on average throughout the different studies, this implies approximately a $0.533 \mathrm{~kg}$ higher piglet weight at the end of the nursery period. Under field conditions, each additional $\mathrm{kg}$ of piglet weight during the nursery period is considered to result in at least $2-3 \mathrm{~kg}$ supplementary weight during the fattening period. This implies earlier slaughter at the same weight or heavier fattening pigs at the same slaughter age. Both scenarios mean economic benefit to the swine farmer. Unfortunately, in the current field studies, we were not able to continue following the piglet performances during the fattening phase, due to some farmspecific management scenarios and several practical restrictions.

Nevertheless, the current analysis revealed a major impact of vaccination against PWD due to F4-ETEC and F18-ETEC on the number of days under antimicrobial treatment, as indicated by the significant reduction in TI100 in the Vaccine-treated group (2.68 \pm 1.69 days) as compared to the Control group (17.74 \pm 4.95 days). Combined with at least an equal to non-significantly higher ADWG, this may be considered as a major achievement, especially since in practice many swine farmers and veterinarians fear reduced piglet performance when group treatments with antimicrobials are reduced 
or abolished, and replaced by individual treatments only. With the recent decisions of the European Parliament and of the Council (Regulation 2019/6 of 11th December 2018 [28]) in mind, reduction in antimicrobial use will become a hot topic within the European Union in the near future. In Article 107 of EU Regulation 2019/6, it is clearly stated that (1) antimicrobial medicinal products shall not be applied routinely, nor used to compensate for poor hygiene, inadequate animal husbandry or lack of care or to compensate for poor farm management; and (2) antimicrobial medicinal products shall not be used for prophylaxis other than in exceptional cases for the administration to an individual animal or a restricted number of animals when the risk of an infection or an infectious disease is very high and the consequences are likely to be severe [28]. The current study clearly demonstrated that this farmer's perception is incorrect and reduction in antimicrobial use, combined with appropriate preventive measures such as vaccination against the specific pathogens involved, may result in equal piglet performances.

Recently, vaccination with a live non-pathogenic E. coli F4 or E. coli F4/F18 vaccine has demonstrated efficacy against PWD due to F4-ETEC, and F4-ETEC and F18-ETEC [26,27]. Immunization against the F4-ETEC and F18-ETEC pathogens resulted in decreased severity and duration of PWD clinical signs and fecal shedding of F4-ETEC and F18-ETEC [26,27]. Moreover, increased weight gain was demonstrated in piglets vaccinated with an $E$. coli $\mathrm{F} 4 / \mathrm{F} 18$ vaccine $[26,29,30]$. The current field results are in line with these observations, indicating that different farm management practices (BMS, weaning age, feeding regime) had no impact on results induced by immunization with an E. coli F4/F18 vaccine under field conditions. This implies that farms suffering from PWD due to F4-ETEC or F18ETEC do not have to alter their specific feeding strategy. This is an advantage, since in most cases there are limitations in the number of available feed bins for the on-farm post-weaning facilities. From an economic point of view, however, 3-phase feeding strategies have been shown to provide optimal performance parameters related to FCR [30].

Another important evaluation parameter to assess the success of different intervention strategies in relation to PWD due to E. coli is mortality [26]. Mortality data were significantly different between treatment groups (5.42 and $2.33 \%$ in Control and Vaccine-treated groups, respectively). These results are in accordance with previous studies $[26,27,29,30]$, which all demonstrated a reduction in mortality following vaccination with an oral live non-pathogenic E. coli F4/F18 vaccine.

\section{Conclusion}

In conclusion, the present study demonstrated the efficacy of an oral live non-pathogenic E. coli F4/F18 vaccine (Coliprotec F4/ F18; Elanco) for active immunization of piglets against PWD due to F4-ETEC and F18-ETEC. In several economically important performance parameters, such as FCR, mortality rate and TI100, E. coli F/F18 vaccination performed significantly better as compared to the standard therapeutic approach. Therefore, vaccination against PWD due to F4-ETEC or F18-ETEC using an oral live non-pathogenic E. coli F4/F18 vaccine may be considered a good alternative to consolidate post-weaning piglet performance results while meeting the new European requirements concerning prudent use of antimicrobials in intensive pig production.

\section{Declarations}

Ethics approval and consent to participate: Field trials with Veterinary Medicinal Product approved for use in swine. No additional ethical approval needed. Consent to participate was obtained following full information of the farmers on the protocol to be carried out.

Funding: The study was funded by Elanco, which facilitated the conduct of the field trials.

Author's contributions: FV coordinated the entire study from study design to data collection and analysis to the manuscript.

\section{Acknowledgements}

The authors greatly acknowledge the different swine farmers and their swine veterinarians for their assistance in preparation, randomization, weighing and data collection during the field trials.

Author's information: FV is currently a Principal Technical Consultant Swine for Benelux/UK\&ROI within Elanco. He holds a DVM, a Master in Veterinary Public Health and Food Safety, a PhD in Veterinary Sciences and a PhD in Applied Biological Sciences, and he has a specific interest in swine intestinal health and the specific approach to improve intestinal health through non-antimicrobial solutions.

\section{References}

1. Fairbrother JM, Nadeau É, Gyles CL. Escherichia coli in postweaning diarrhoea in pigs: an update on bacterial types, pathogenesis, and prevention strategies. Anim Heal Res Rev. 2005; 6: 17-39.

2. Hoa NX, Kalhoro DH, Lu C. Distribution of serogroups and virulence genes of $E$. coli strains isolated from porcine post weaning diarrhoea in Thua Thien Hue province Vietnam. Tạp chí Công ngh Sinh học. 2013; 11: 665-672.

3. Lyutskanov M. Epidemiological characteristics of post-weaning diarrhoea associated with toxin-producing Escherichia coli in large intensive pig farms. Trakia J Sci. 2011; 9: 68-73.

4. Svensmark B, Jorsal SE, Nielsen K, Willeberg P. Epidemiological studies of piglet diarrhoea in intensively managed Danish sow herds. I. Pre-weaning diarrhoea. Acta Vet Scand. 1989; 30: 43-53.

5. Svensmark B, Nielsen K, Willeberg P, Jorsal SE. Epidemiological studies of piglet diarrhoea in intensively managed Danish sow herds. II. Post-weaning diarrhoea. Acta Vet Scand. 1989; 30: 55-62.

6. Tubbs RC, Hurd HS, Dargatz D, Hill G. Preweaning morbidity and mortality in the United States swine herd. Swine Heal Prod. 1993; 1: 21-28.

7. USDA. Part II. Reference of Swine Health and Health Management in the United States, 2000. USDA:APHIS:VS, CEAH, National Animal Health Monitoring System, Fort Collins, CO. 2002

8. Zhang W, Zhao M, Ruesch L, Omot A, Francis D. Prevalence of virulence genes in Escherichia coli strains recently isolated from young pigs with diarrhoea in the US. Vet Microbiol. 2007; 123: 145-152.

9. Luppi A, Gibellini M, Gin T, Vangroenweghe F, Vandenbroucke V, Bauerfeind $\mathrm{R}$, et al. Prevalence of virulence factors in enterotoxigenic Escherichia coli isolated from pigs with post-weaning diarrhoea in Europe. Porcine Health Manag. 2016; 2: 20-25.

10. Vangroenweghe $F$, Luppi $A$, Thas $O$. Prevalence of enterotoxigenic Escherichia coli pathotypes and virotypes isolated from piglets suffering from post-weaning diarrhea in Belgium and the Netherlands. Arch Vet Anim Sci. 2020; 2: 1-8.

11. Fairbrother JM, Gyles CL. Chapter 53: Colibacillosis. In: Diseases of Swine. $10^{\text {th }}$ Edition. Eds. Zimmerman JJ, Karriker LA, Ramirez A, Schwartz KJ, 
Stevenson GW. Wiley-Blackwell. 2012; 723-749.

12. Chen X, Gao S, Jiao X, Liu XF. Prevalence of serogroups and virulence factors of Escherichia coli strains isolated from pigs with postweaning diarrhoea in eastern China. Vet Microbiol. 2004; 103: 13-20.

13. Frydendahl K. Prevalence of serogroups and virulence genes in Escherichia coli associated with postweaning diarrhoea and edema disease in pigs and a comparison of diagnostic approaches. Vet Microbiol. 2002; 85: 169-182.

14. Vu-Khac H, Holoda E, Pilipcinec E, Blanco M, Blanco JE, Mora A, et al. Serotypes, virulence genes, and PFGE profiles of Escherichia coli isolated from pigs with postweaning diarrhoea in Slovakia. BMC Vet Res. 2006; 2 13-20.

15. Abraham S, Trott DJ, Jordan D, Gordon DM, Groves MD, Fairbrother JM et al. Phylogenetic and molecular insights into the evolution of multidrugresistant porcine enterotoxigenic Escherichia coli in Australia. Int J Antimicrob Agents. 2014; 44: 105-111.

16. Abraham S, Jordan D, Wong HS, Johnson JR, Toleman MA, Wakeham $\mathrm{DL}$, et al. First detection of extended-spectrum cephalosporin- and fluoroquinoloneresistant Escherichia coli in Australian food-producing animals. J Glob Antimicrob Resist. 2015; 3: 273-277.

17. Boyen F, Vangroenweghe F, Butaye P, De Graef E, Castryck F, Heylen P et al. Disk prediffusion is a reliable method for testing colistin susceptibility in porcine E. coli strains. Vet Microbiol. 2010; 144: 359-362.

18. Jahanbakhsh S, Smith MG, Kohan-Ghadr HR, Letellier A, Abraham S Trott DJ, et al. Dynamics of extended-spectrum cephalosporin resistance in pathogenic Escherichia coli isolated from diseased pigs in Quebec, Canada. Int J Antimicrob Agents. 2016; 48: 194-202.

19. Luppi A, Bonilauri P, Dottori M, Gherpelli Y, Biasi G, Merialdi G, et al Antimicrobial resistance of $\mathrm{F}^{+}$Escherichia coli isolated from swine in Italy. Transbound Emerg Dis. 2013; 62: 67-71.

20. Jha R, Berrocoso JD. Review: dietary fiber utilization and its effects on physiological functions and gut health of swine. Animal. 2015; 9: 1441-1452.
21. Jha R, Berrocoso JFD Dietary fiber and protein fermentation in the intestine of swine and their interactive effects on gut health and on the environment: $a$ review. Anim Feed Sci Technol. 2016; 212: 18-26.

22. Tran THT, Everaert N, Bindelle J. Review on the effects of potential prebiotics on controlling intestinal enteropathogens Salmonella and Escherichia coli in pig production. J Anim Physiol Anim Nutr (Berl). 2018; 102: 17-32.

23. Poulsen HD. Zinc oxide for weanling pigs. Acta Agric Scand. 1995; 45: 159165.

24. European Medicinal Agency. Questions and answers on veterinary medicinal products containing zinc oxide to be administered orally to food producing species. Outcome of a referral procedure under Article 35 of Directive 2001/82/EC (EMEA/V/A/118). 2017; EMA/394961/2017.

25. Melkebeek V, Goddeeris BM, Cox E. ETEC vaccination in pigs. Vet Immunol Immunopathol. 2013; 152: 37-42.

26. Fairbrother JM, Nadeau E, Bélanger L, Tremblay C-L, Tremblay D, Brunelle $\mathrm{M}$, et al. Immunogenicity and protective efficacy of a single-dose live nonpathogenic Escherichia coli oral vaccine against F4-positive enterotoxigenic Escherichia coli challenge in pigs. Vaccine. 2017; 35: 353-360.

27. Nadeau E, Fairbrother JM, Zentek J, Bélanger L, Tremblay D, Tremblay C-L, et al. Efficacy of a single oral dose of a live bivalent $E$. coli vaccine against post-weaning diarrhoea due to F4 and F18-positive enterotoxigenic E. coli. Vet J. 2017; 226: 32-39.

28. Regulation (EU) 2019/6 of the European Parliament and of the Council of 11 December 2018 on veterinary medicinal products and repealing Directive 2001/82/EC. Official Journal of the European Union L. 4/43.

29. Vangroenweghe $F$, Thas $O$. Improved piglet performance and reduced antibiotic use following oral vaccination with a live avirulent J Clin Res Med. 2020a; 3: 1-8.

30. Vangroenweghe $\mathrm{F}$, Thas $\mathrm{O}$. Application of high energy and protein ciets in combination with a live avirulent Escherichia coli $\mathrm{F} 4$ vaccine against postweaning diarrhea. Vacc Res. 2020b; 7: 1-9. 